\section{New approach to colorectal cancer screening could avoid unnecessary colonoscopy}

The Guaiac fecal occult blood test (FOBT) for colorectal cancer has low sensitivity and specificity and can lead to unnecessary colonoscopy. A recent observational study by Fraser and coworkers indicates that a reflex two-tier approach to colorectal cancer screening, whereby individuals with positive guaiac FOBT results proceed to immunochemical FOBTs, can identify those at high risk for colorectal malignancy and select those most likely to benefit from colonoscopy.

This study is the first analysis of the two-tier approach and was carried out during the second round of screening for colorectal cancer in a pilot study in Scotland, UK. Patients aged 5069 years with a positive guaiac FOBT result provided two fecal samples for immunochemical FOBTs while awaiting colonoscopy. Samples from 800 participants were then classified as being both negative (N/N;22\%), one negative and one positive (N/P; 16\%), or both positive (P/P; 62\%). All participants then underwent colonoscopy. Colorectal cancer was detected in $<1 \%$ of those with an N/N or N/P immunochemical FOBT result, compared with $8 \%$ of individuals with a $\mathrm{P} / \mathrm{P}$ result. In $\mathrm{P} / \mathrm{P}$ individuals, odds ratios for invasive cancer, all adenomatous polyps, and large $(>10 \mathrm{~mm})$ adenomatous polyps were $7.57,1.48$, and 3.11 , respectively. Immunochemical FOBT sensitivity and specificity for colorectal cancer were $95 \%$ and $39.5 \%$, respectively. In summary, colonoscopy could be avoided in guaiac-FOBT-positive individuals with a negative immunochemical FOBT result, which could reduce the costs and morbidity associated with colonoscopy in colorectal cancer screening programs.

Alexandra King

Original article Fraser CG et al. (2006) Immunochemical testing of individuals positive for guaiac faecal occult blood test in a screening programme for colorectal cancer: an observational study. Lancet Oncol 7: 127-131

\section{Protein biomarker signatures for accurate diagnosis of urothelial cancer}

Urothelial cancer can be diagnosed by identification of molecular changes associated with disease pathogenesis, but this approach can lack specificity and sensitivity, possibly because it doesn't include enough appropriate diseasespecific markers. Theodorescu et al. used molecular techniques to identify a DISEASE-PROTEOME 'SIGNATURE' that could distinguish urothelial cancer from nonmalignant urological disorders and other genitourinary cancers in urine samples.

POLYPEPTIDES isolated from urine samples of patients with urothelial cancer $(n=46)$ or healthy volunteers $(n=33)$ were analyzed using MASS SPECTROMETRY. The mass patterns obtained were used to develop a framework for identifying the cancer, and this model was subsequently improved by analysis of urine samples $(n=366)$ from patients with malignant or nonmalignant genitourinary disease and from healthy individuals.

A panel of 22 polypeptides was identified that could distinguish between samples from patients with urothelial carcinoma and those from healthy volunteers. A blind analysis showed that this pattern was sensitive and specific, identifying 100\% (95\% Cl $87-100 \%)$ of urothelial carcinoma cases and $100 \%$ (95\% Cl $84-100 \%)$ of healthy cases. Furthermore, the proteomic signature could be used to distinguish between urothelial cancer and other genitourinary diseases, with a correct classification range of $86-100 \%$.

Bioinformatic tools for analyzing diseasespecific patterns of polypeptides in urine could be used for the diagnosis of a range of urological disorders and could reduce the occurrence of false-positive diagnoses. Further studies are in progress to test whether this approach can be used to predict tumor behavior and response to treatment in patients with bladder cancer.

Kate Matthews

Original article Theodorescu D et al. (2006) Discovery and validation of new protein biomarkers for urothelial cancer: a prospective analysis. Lancet Oncol 7: 230-240

\section{Does BPSO reduce mortality in patients with BRCA mutations?}

Bilateral prophylactic salpingo-oophorectomy (BPSO) has been shown to reduce the risks of breast and ovarian cancer, and is often performed in women with BRCA1 or BRCA2 gene mutations. Whether BPSO actually reduces mortality in patients with these mutations, however, is unclear, and is the subject of a study reported in Lancet Oncology.
GLOSSARY

DISEASE-PROTEOME 'SIGNATURE'

The expression pattern of a complete set of proteins in a disease

POLYPEPTIDES

Chains of amino acids linked by covalent bonds that join to form proteins

MASS SPECTROMETRY A technique that identifies components of a molecule according to their molecular mass and charge 